\title{
Intraperitoneal EpCAM-Targeted Immunotoxin: A First Step Towards Engineering the Immune Environment in Colorectal Peritoneal Metastases?
}

\author{
Wim Ceelen, MD, PhD, FACS, FSSO \\ Department of GI Surgery, Ghent University Hospital, Ghent, Belgium
}

In this issue of the journal, Thorgersen and coworkers report peritoneal and systemic cytokine response after cytoreductive surgery and hyperthermic intraperitoneal chemoperfusion (HIPEC) with or without adjuvant intraperitoneal instillation, the morning after surgery, of the immunotoxin MOC31PE. ${ }^{1}$ Immunotoxins (ITs) are bifunctional proteins composed of a targeting antibody linked to a complete or modified protein toxin. ${ }^{2}$ MOC31PE is a monoclonal mouse IgG1 targeting the epithelial cell adhesion molecule (EpCAM, also known as CD326 or MOC31; Fig. 1) developed by a collaborative group at the Norwegian Radium Hospital. EpCAM is a cell surface transmembrane protein typically expressed in embryonic cells and in adult epithelial tissues, where it is restricted to the basolateral domain. ${ }^{3}$ Its role as a cancer antigen was first identified in colorectal cancer (CRC), and EpCAM has been implicated in several features of malignant growth, including proliferation, migration, stemness, and epithelialto-mesenchymal transition (EMT). ${ }^{4}$ The monoclonal antibody is covalently linked with the intact Pseudomonas aeruginosa exotoxin A (PE). Upon binding of MOC31PE with EpCAM, the bound receptor is internalized, and the PE cleaved off, processed, and released in the cytosol, where it inhibits elongation factor-2 and thereby blocks elongation of polypeptides, which eventually leads to cell death by apoptosis.

(C) Society of Surgical Oncology 2021

First Received: 31 March 2021

Accepted: 1 May 2021;

Published Online: 15 May 2021

W. Ceelen, MD, PhD, FACS, FSSO

e-mail: Wim.ceelen@ugent.be
Due to their bacterial origin, immunotoxins are highly immunogenic and elicit the production of antibodies, which render subsequent therapy less effective. For that reason, a phase I trial of systemic MOC31PE in cancer patients was performed in cohorts with and without oral administration of cyclosporin, which was shown to delay the generation of anti-immunotoxin antibodies. ${ }^{5}$ The same group later showed, rather unexpectedly, that survival of patients included in the phase I trial was significantly better in those who did not receive cyclosporin; the magnitude of the difference in survival was especially impressive in CRC (16 versus 6 months). Interestingly, analysis of serum samples from patients in both groups showed a significantly higher release of Th1-related cytokines, including interleukin (IL)-2, IL-12, interferon (IFN)- $\gamma$, tumor necrosis factor (TNF)- $\alpha$, IL-1 $\beta$, and granulocyte-macrophage colony-stimulating factor (GM-CSF), in the MOC31PE alone group. In vitro, the authors showed induction of immunogenic cell death (ICD) upon exposure of HCT116 and SW480 cell lines to MOC31PE; at the same time, maturation of dendritic cells was observed.

Based on these results, a phase I trial (ImmunoPeCa, NCT00769405) was initiated at the same institution, testing IP administration in the setting of minimal residual disease, after cytoreductive surgery (CRS) and HIPEC with mitomycin C for EpCAM-positive colorectal cancer. ${ }^{7}$ No doselimiting toxicity was observed, and systemic absorption of MOC31PE was minimal. However, all patients developed neutralizing antibodies. Analysis of long-term outcome, which included an additional expansion cohort resulting in a total of 21 patients, showed promising results, with median overall survival that was not reached (estimated 3 -year survival of $78 \%$ ) and a median disease-free survival of $33 \% .^{8}$ 


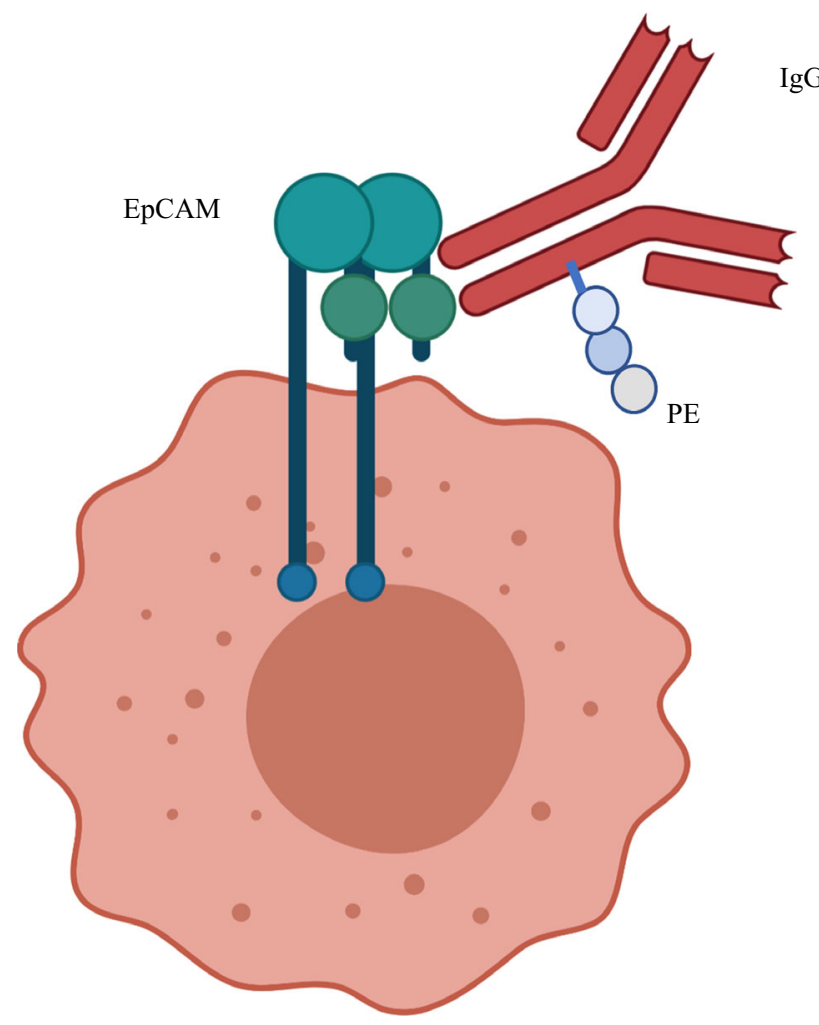

FIG. 1. MOC 31PE consists of a monoclonal IgG directed against the epithelial cell adhesion molecule (EpCAM), a cell surface transmembrane protein highly expressed by colorectal cancer cells. The antibody is covalently linked to Pseudomonas aeruginosa exotoxin $\mathrm{E}(\mathrm{PE})$

The present study reports the analysis of cytokines in peritoneal and plasma samples from 12 patients who received the highest dose level in the ImmunoPeCa trial $(10 \mu \mathrm{g} / \mathrm{kg})$, as compared with 26 patients who underwent CRS and HIPEC alone. ${ }^{1}$ Analyses were performed at five timepoints during three successive postoperative days. Of note, the peritoneal disease burden was low in both groups (median peritoneal cancer index 7 and 5 in treatment and control group, respectively), and while the experimental group only consisted of CRC, $31 \%$ of patients in the control group had pseudomyxoma peritonei. Only modest effects were noted in the systemic compartment, but effluents from the postoperative peritoneal cavity showed striking differences in cytokine release between the two groups. Specifically, adjuvant MOC31PE treatment led to significantly higher peritoneal concentrations of cytokines IL-1 $\beta$, TNF- $\alpha$, IL-6, and IL-12, chemokines monocyte chemotactic protein (MCP)-1, macrophage inflammatory protein (MIP)- $1 \alpha$, and MIP-1 $\beta$, and growth factors granulocyte colony-stimulating factor (G-CSF) and IL-7. No differences were observed in vascular endothelial growth factor (VEGF), fibroblast growth factor (FGF), IL-2, IL-4, IL-9, or IL-13. Interestingly, peritoneal IFN- $\gamma$ concentration increased over time in the experimental group, but decreased in the control group. Since presumably the mass of residual colorectal cancer cells is minimal at time of administration of MOC31PE, and normal mesothelial cells do not express EpCAM, the observed inflammatory response is likely explained by direct effects of the exotoxin on peritoneal inflammatory and immune cell populations.

It is tempting to hypothesize that the proinflammatory environment elicited by IP administration of MOC31PE can enhance antitumor immunity and maybe explain the promising overall and disease-free survival results observed in the phase I trial. In preclinical models of malignant mesothelioma, PE-based immunotoxins did indeed increase tumor infiltration of CD8+ cells, and acted synergistically with immune checkpoint inhibitors. ${ }^{9}$ On the other hand, some of the cytokines released to a greater extent in the experimental group (IL-6, IL-10, and TNF- $\alpha$ ) have been implicated in postoperative immune suppression. ${ }^{10}$ Clearly, elucidation of the relationship between the observed inflammatory changes and the function of the immune system after IP delivery of MOC31PE is a priority for further preclinical and translational research.

The treatment of colorectal peritoneal metastases remains a challenge: the majority of patients will develop recurrent peritoneal cancer, and recent trials have called into question the added value of HIPEC with oxaliplatin. The results of the ImmunoPeCa trial illustrate the increasing recognition of the importance of the peritoneal (immune) microenvironment in determining therapy response. ${ }^{11}$ In the era of immune therapy, surprisingly little is known on the postoperative peritoneal host defense, despite the relative ease of obtaining surgical samples and drain fluids for analysis. ${ }^{12}$ In animal models, several approaches including CAR-T cell therapy and oncolytic virotherapy have demonstrated the potential to reverse the immunosuppressive tumor microenvironment in colorectal PM. ${ }^{13,14}$ However, clinical translation is at present virtually nonexistent. The only approved intraperitoneal immune therapy was the chimeric antibody catumaxomab (Removab), which targets EpCAM and CD3. ${ }^{15}$ However, the product has been withdrawn from the market for commercial reasons.

In conclusion, the work of Thorgersen and the overall results of the ImmunoPeCa trial suggest the potential of engineering the peritoneal immune environment in patients with colorectal peritoneal metastases. ${ }^{1}$ Additional mechanistic insights and results from comparative clinical trials will allow the place of immunotoxins in the therapeutic armamentarium to be determined for these patients.

DISCLOSURES I have no conflicts of interest to declare. 


\section{REFERENCES}

1. Thorgersen EB, Asvall J, Frøysnes IS, et al. Increased local inflammatory response to MOC31PE immunotoxin after cytoreductive surgery and hyperthermic intraperitoneal chemotherapy. Ann Surg Oncol. 2021. https://doi.org/10.1245/s10434-021-1002 2-0.

2. Kim JS, Jun SY, Kim YS. Critical issues in the development of immunotoxins for anticancer therapy. $J$ Pharm Sci. 2020;109(1):104-15.

3. Fagotto F, Aslemarz A. EpCAM cellular functions in adhesion and migration, and potential impact on invasion: a critical review. Biochim Biophys Acta Rev Cancer. 2020;1874(2):188436.

4. Gires O, Pan M, Schinke H, Canis M, Baeuerle PA. Expression and function of epithelial cell adhesion molecule EpCAM: where are we after 40 years? Cancer Metastasis Rev. 2020;39(3):969-87.

5. Andersson Y, Engebraaten O, Juell S, et al. Phase I trial of EpCAM-targeting immunotoxin MOC31PE, alone and in combination with cyclosporin. Br J Cancer. 2015;113(11):1548-55.

6. Andersson Y, Inderberg EM, Kvalheim G, et al. Immune stimulatory effect of anti-EpCAM immunotoxin-improved overall survival of metastatic colorectal cancer patients. Acta Oncol. 2020;59(4):404-9.

7. Frøysnes IS, Andersson Y, Larsen SG, et al. Novel treatment with intraperitoneal MOC31PE immunotoxin in colorectal peritoneal metastasis: results from the ImmunoPeCa phase 1 trial. Ann Surg Oncol. 2017;24(7):1916-22.

8. Frøysnes IS, Andersson Y, Larsen SG, et al. ImmunoPeCa trial: long-term outcome following intraperitoneal MOC31PE immunotoxin treatment in colorectal peritoneal metastasis. Eur $J$ Surg Oncol. 2021;47(1):134-8.

9. Leshem Y, O’Brien J, Liu X, et al. Combining local immunotoxins targeting mesothelin with CTLA-4 blockade synergistically eradicates murine cancer by promoting anticancer immunity. Cancer Immunol Res. 2017;5(8):685-94.

10. Bakos O, Lawson C, Rouleau S, Tai LH. Combining surgery and immunotherapy: turning an immunosuppressive effect into a therapeutic opportunity. J Immunother Cancer. 2018;6(1):86.

11. Ceelen W, Ramsay RG, Narasimhan V, Heriot AG, De Wever O. Targeting the tumor microenvironment in colorectal peritoneal metastases. Trends Cancer. 2020;6(3):236-46.

12. Franko J, Brahmbhatt R, Tee M, et al. Cellular immunoprofile of peritoneal environment during a HIPEC procedure. Ann Surg Oncol. 2020;27(13):5005-13.

13. Liu Z, Ravindranathan R, Kalinski P, Guo ZS, Bartlett DL. Rational combination of oncolytic vaccinia virus and PD-L1 blockade works synergistically to enhance therapeutic efficacy. Nat Commun. 2017;8:14754.

14. Thadi A, Khalili M, Morano WF, Richard SD, Katz SC, Bowne WB. Early investigations and recent advances in intraperitoneal immunotherapy for peritoneal metastasis. Vaccines (Basel). 2018;6(3):54

15. Ströhlein MA, Lordick F, Rüttinger D, et al. Immunotherapy of peritoneal carcinomatosis with the antibody catumaxomab in colon, gastric, or pancreatic cancer: an open-label, multicenter, phase I/II trial. Onkologie. 2011;34(3):101-8.

Publisher's Note Springer Nature remains neutral with regard to jurisdictional claims in published maps and institutional affiliations. 